\title{
AN AXIOM FOR NONSEPARABLE BOREL THEORY ${ }^{1}$ \\ BY
}

WILLIAM G. FLEISSNER

\begin{abstract}
Kuratowski asked whether the Lebesgue-Hausdorff theorem held for metrizable spaces. A. Stone asked whether a Borel isomorphism between metrizable spaces must be a generalized homeomorphism. The existence of a $Q$ set refutes the generalized Lebesgue-Hausdorff theorem. In this paper we discuss the consequences of the axiom of the title, among which are "yes" answers to both Kuratowski's and Stone's questions. The axiom states that a point finite analytic additive family is $\sigma$ discretely decomposable. We show that this axiom is valid in the model constructed by collapsing a supercompact cardinal to $\omega_{2}$ using Lévy forcing. Our proof displays relationships between $\sigma$ discretely decomposable families, analytic additive families and $d$ families.
\end{abstract}

1. Introduction. Several fields of mathematics have roots in the theory of a real variable, which flourished at the end of the nineteenth century and the beginning of the twentieth century. At this time, investigation centered on complete separable metric spaces and their metrizable continuous images. As mathematicians became interested in abstract topological spaces, it was natural to ask whether classical theorems in the theory of a real variable generalized to a wider class of spaces. Indeed, Kuratowski explicitly asked as early as 1935 [11], whether certain theorems of Baire, Lebesgue, Hausdorff, and Banach held for all metrizable spaces. More recently, A. Stone began an investigation of the Borel theory of metrizable spaces. Because this paper centers on such questions, we make the convention that all spaces considered are metrizable. (The exceptions, clear from context, are in remarks in \$4.) Stone left open the important question of whether a Borel isomorphism must be a generalized homeomorphism. A one-to-one function $f$ is a Borel isomorphism if both $f$ and $f^{-1}$ take open sets to Borel sets; $f$ is a generalized homeomorphism of type $(\alpha, \beta)$ if $f$ takes open sets to $\Sigma_{\beta}$ sets and $f^{-1}$ takes open sets to $\Sigma_{\alpha}$ sets. $\left(\Sigma_{\alpha}\right.$ is the family of Borel sets of additive class $\alpha ; \Pi_{\alpha}$ is the family of Borel sets of multiplicative class $\alpha ; \alpha, \beta<\omega_{1}$.)

Significant progress on these questions was made by Stone's student

Received by the editors December 12, 1977 and, in revised form, May 16, 1978.

AMS (MOS) subject classifications (1970). Primary 02K05, 04A15, 26A21; Secondary 02K35.

$K e y$ words and phrases. Borel mapping, Baire function, $\sigma$ discretely decomposable, analytically additive, $d$ family, decompositions of metrizable spaces, $Q$ sets, Lévy forcing, $\diamond, E(\kappa)$, supercompact cardinals, Borel isomorphism, generalized homeomorphism.

${ }^{1}$ Partially supported by NSF grant MCS 78-09484. 
Hanseil [5], [6]. Hansell defined $\sigma$ discrete decompositions, and showed that much of the theory for separable spaces and complete spaces carries over to $\sigma$ discrete functions. That for absolutely analytic spaces the functions in question are $\sigma$ discrete follows from

1.1. HANSELl's TheOREM [5]. A disjoint, absolutely analytic, additive family is $\sigma$ discretely decomposable.

A set is analytic in a space if it can be obtained from the open sets by the $\mathbb{Q}$ operation. A set is absolutely analytic if it is analytic in every superspace, or, equivalently, in one completion. Let $P$ be a property of sets, such as absolutely analytic. A family of sets is $P$ additive if the union of every subfamily has property P. A family of subsets in a space is discrete if every point of the space has a neighborhood meeting at most one element of the family. A family $\mathcal{S}=\left\{S_{i}: i \in I\right\}$ is $\sigma$ discretely decomposable $(\sigma \mathrm{dd})$ if there is a family $\mathcal{S}^{\prime}=\left\{S_{i, n}: i \in I, n \in \omega\right\}$, a $\sigma$ discrete decomposition of $\mathcal{S}$, satisfying

$$
\begin{aligned}
& \text { for all } i \in I, \quad S_{i}=\cup\left\{S_{i, n}: n \in \omega\right\}, \\
& \text { for all } n \in \omega, \quad\left\{S_{i, n}: i \in I\right\} \text { is a discrete family. }
\end{aligned}
$$

Since each $x \in S_{i, n}$ has a neighborhood meeting only $S_{i, n}$ of $\left\{S_{j, n}: j \in I\right\}$,

for all $i \in I, n \in \omega$ there is an open set $U_{n}^{i}$, such that $S_{i, n}=S_{i} \cap U_{n}^{i}$.

A function $f: X \rightarrow Y$ is $\sigma$ discrete if there is a base $\mathscr{B}$ for $Y$ such that $\left\{f^{\leftarrow B}\right.$ : $B \in \mathscr{B}\}$ is $\sigma$ dd, where

$$
f^{\leftarrow} B=\{x \in X: f(x) \in B\} .
$$

With the advent of set theoretic consistency results in the 1960's, it became clear that Kuratowski's question could not be answered "yes" in ZFC, the usual axioms of set theory. Define a $Q$ set to be an uncountable subset $X$ of the real line such that every subset of $X$ is a relative $F_{\alpha}\left(\right.$ i.e., $\left.\mathscr{P}(X)=\Sigma_{1} X\right)$. Martin and Solovay [13] showed that the existence of a $Q$ set followed from Martin's axiom and the negation of the Continuum Hypothesis, which Solovay and Tennenbaum [23] showed consistent with ZFC. We will show below that a $Q$ set gives a counterexample to the generalized LebesgueHausdorff theorem.

In this paper we complement the $Q$ set result by showing consistent an axiom, Proposition $\mathbf{P}$, which answers Kuratowski's and Stone's questions "yes" To be more precise, we show that Proposition $\mathrm{P}$ is consistent modulo a large cardinal axiom; by standard techniques our proof can by cast in the form (CON ZFC $+\exists$ a supercompact cardinal) $\rightarrow($ CON ZFC + Proposition P). Hansell [5], [6] implicitly proposed Proposition P (with "point finite" replaced by "disjoint"). Indeed, Hansell's theorem is a special case. 
Proposition P is simply a yes answer to Question 3 of Kaniewski and Pol [10]. Call a family point finite if every point is a member of at most finitely many elements of the family.

Proposition P. A point finite analytic additive family is $\sigma$ discretely decomposable.

This paper is organized as follows. In the next section we state consequences of Proposition P. The generalized Baire-Lebesgue-Hausdorff, Banach, and Lavrentiev theorems are due to Hansell. One simply modifies Hansell's proofs [5], [6] by replacing the application of Hansell's theorem with the assumption of Proposition P. The selection theorems of Kaniewski and Pol [10] follow similarly. We do not know to whom to attribute the result that Proposition P implies that a Borel isomorphism is a generalized homeomorphism.

In $\$ 3$ we discuss properties that a family of sets might have and discuss the implications between these properties with various extra axioms of set theory. We sketch the proof of Proposition P from certain other axioms. The proof is broken down into a number of lemmas, which we prove in $\$ 4$.

In the final section we discuss the model constructed by using Lévy forcing to collapse a supercompact cardinal to $\omega_{2}$. We show that the axioms used in $\$ 4$ are valid in this model.

The author wishes to express thanks for fruitful conversations with Roman Pol, Eric van Douwen, Peter Nykios, John Jayne, and Arthur Stone. He gives special thanks to Bill Weiss for carefully reading a preliminary version and insisting that hand waving be replaced by proofs.

2. Consequences of Proposition P. The first part of this section lists consequences of Proposition $\mathrm{P}$, which we mark with $(\mathrm{P})$ to indicate the assumption of Proposition P. The second part gives counterexamples derived from other extra axioms of set theory and discusses open questions.

As we indicated in the Introduction, Theorems 2.1-2.4 are implicit in Hansell and Theorem 2.5 in Kaniewski and Pol. Theorems 2.1, 2.2 and 2.5 are generalizations in that the hypothesis that $Y$ be separable is omitted. Theorems 2.3 and 2.4 are generalizations in that $f$ is allowed to be a generalized homeomorphism rather than a homeomorphism.

2.1a. Generalized Baire, Lebesgue-Hausdorff Theorem [1], [9], [7]. $(P)$. Let $X$ be a space and $Y$ a Banach space. There is a level-by-level correspondence between the hierarchy of Baire functions and the hierarchy of Borel measurable functions. Moreover these functions are continuous when restricted to a residual set.

A residual set is the complement of a first category set. To express more 
precisely the correspondence between the Borel measurable hierarchy, $\left\{\Psi_{\alpha} X Y: \alpha<\omega_{1}\right\}$, and the Baire hierarchy, $\left\{\Phi_{\alpha} X Y: \alpha<\omega_{1}\right\}$, we define

$$
\Psi_{\alpha} X Y=\left\{f:\left(\forall U \in \Sigma_{0} Y\right)\left(f^{\leftarrow} U \in \Sigma_{\alpha} X\right)\right\},
$$

or more concisely,

$$
\begin{gathered}
\Psi_{\alpha} X Y=\left\{f: f^{\leftarrow} \Sigma_{0} Y \subset \Sigma_{\alpha} X\right\}, \\
\Phi_{0} X Y=\Psi_{0} X Y, \\
\Phi_{\alpha} X Y=\left(\cup\left\{\Phi_{\beta}: \beta<\alpha\right\}\right)_{\lambda} \text { for } \alpha>0,
\end{gathered}
$$

where in general $\Delta_{\lambda}$ denotes the set of all functions which are limits of convergent sequences of functions belonging to the class $\Delta$. To obtain an analogue of the Lebesgue-Hausdorff theorem for arbitrary $Y$, Banach [2] defined.

$$
\begin{gathered}
\Phi_{0}^{*} X Y=\Psi_{0} X Y, \quad \Phi_{1}^{*} X Y=\Psi_{1} X Y, \\
\Phi_{\alpha}^{*} X Y=\left(\cup\left\{\Phi_{\beta}^{*}: \beta<\alpha\right\}\right)_{\lambda} \text { for } \alpha>1 .
\end{gathered}
$$

2.1b. Generalized Baire, Lebesgue-Hausdorff Theorem. ( $P$ ). Let $X$ be a space and $Y$ a Banach space. Then (a) and (b) are equivalent and imply (c):

(a) $f \in \Psi_{\alpha} X Y$,

(b) $f \in \Phi_{\alpha} X Y$ if $\alpha$ is finite, $f \in \Phi_{\alpha+1} X Y$ if $\alpha$ is infinite.

(c) $f: X \rightarrow Y$ and there is a residual set $T$ such that $f \mid T$ is continuous. Further, if $\alpha=1, T$ may be chosen so that $f$ is continuous on $T$.

2.2. Generalized Banach Theorem [2]. (P). As in Theorem 2.1b with $Y$ any space and $\Phi^{*}$ replacing $\Phi$.

2.3. Generalized Lavrentiev Theorem [8]. $(P)$. Let $\alpha$ and $\beta$ be two given nonlimit ordinals. Let $f$ be a generalized homeomorphism of type $(\alpha, \beta)$ between arbitrary subsets of two complete spaces $X, Y$. Then $f$ can be extended to a generalized homeomorphism of type $(\alpha, \beta)$ from a member of $\Pi_{\alpha+\beta+1} X$ to a member of $\Pi_{\beta+\alpha+1} Y$.

2.4. THEOREM. (P). If $X$ is absolutely $\Pi_{\alpha}$ and $f: X \rightarrow Y$ is a generalized homeomorphism of type $(\alpha, \beta)$, then $f \rightarrow X=\{f(x): x \in X\}$ is absolutely $\Pi_{\beta+\alpha}$.

2.5. Generalized Kuratowski and Ryll-Nardzewski Selection THEOREM. (P). Every compact valued map $F: X \rightarrow 2^{Y}$ of lower class $\alpha$, $0<\alpha<\omega_{1}$, admits a selector of class $\alpha$. If $F$ is lower Borel, $F$ admits a Borel selector.

We explain the first sentence of Theorem 2.5. The hypothesis is that if $F$ is a function with domain $X, F(x)$ is a compact subset of $Y$ for all $x \in X$, and $\{x: F(x) \cap U \neq \varnothing\} \in \Sigma_{\alpha} X$ for all $U$ open in $Y$. The conclusion is that there 
is an $f \in \Psi_{\alpha} X Y$ with $f(x) \in F(x)$ for all $x \in X$. The last statement of Theorem 2.5 uses Lemma 2.8, below.

Several applications use Lemma 2.8, a simple consequence of Preiss's theorem, Hansell's converse, and Proposition P.

2.6. Preiss's Theorem [20]. If $\mathcal{S}$ is a disjoint Borel additive family, then there is an $\alpha<\omega_{1}$, such that $\delta \subset \Sigma_{\alpha}$.

Proof. We assume that for all $\alpha<\omega_{1} \delta \not \Sigma_{\alpha}$, and aim for a contradiction. We can find $\omega_{1}$ disjoint subsets $\delta_{\beta}$ of $\mathcal{S}$ such that, for all $\alpha, \beta<\omega_{1}$, $\delta_{\beta} \not \subset \Sigma_{\alpha}$. Because $\delta$ is Borel additive, there is a function $\delta: \omega_{1} \rightarrow \omega_{1}$ so that $\cup \mathcal{S}_{\beta} \subset \Sigma_{\delta(\beta)}$ and $\delta(\beta) \geqslant \beta$. Now choose $S_{\beta} \in \mathcal{S}_{\beta}, S_{\beta} \notin \Sigma_{\delta(\beta)}$. Let $A=$ $\cup\left\{S_{\beta}: \beta<\omega_{1}\right\}$. Then for some $\alpha, A \in \Sigma_{\alpha}$. Now $S_{\alpha}=A \cap \cup \delta_{\alpha} \in \Sigma_{\delta(\alpha)}$. This contradicts our choice of $S_{\alpha}$.

2.7. Hansell's Converse [5]. Suppose $\mathcal{S}=\left\{S_{i}: i \in I\right\}$ is a o discretely decomposable subset of $\Sigma_{\alpha} X$. Then $\mathcal{S}$ is $\Sigma_{\alpha}$ additive. Similarly with $\Sigma_{\alpha}$ replaced by analytic.

Proof. First we note that the union of a discrete family of $\Sigma_{\alpha}$ (respectively, $\Pi_{\alpha}$ ) sets is $\Sigma_{\alpha}$ (respectively, $\Pi_{\alpha}$ ). (This is proved straightforwardly by induction on $\alpha$.)

Let $\left\{S_{i, n}: i \in I, n \in \omega\right\}$ be a $\sigma$ discrete decomposition as in (1.1)-(1.3). $S_{i, n}$, the intersection of a $\Sigma_{\alpha}$ set and an open set, is $\Sigma_{\alpha}$. Let $J$ be an arbitrary subset of $I . S_{n}^{J}=\cup\left\{S_{i, n}: i \in J\right\}$, the discrete union of $\Sigma_{\alpha}$ sets, is $\Sigma_{\alpha}$. Finally

$$
\cup\left\{S_{i}: i \in J\right\}=\cup\left\{S_{n}^{J}: n \in \omega\right\},
$$

the countable union of $\Sigma_{\alpha}$ sets, is $\Sigma_{\alpha}$.

2.8. LEMMA. ( $P$ ). If $\mathcal{S}$ is a disjoint Borel additive family, then there is an $\alpha<\omega_{1}$ such that $\mathcal{S}$ is $\Sigma_{\alpha}$ additive.

2.9. COROllaRY. $(P)$. Borel isomorphisms are generalized homeomorphisms.

Proof. Let $f: X \rightarrow Y$ be a Borel isomorphism. Let $\mathscr{B}=\cup\left\{\mathscr{B}_{m}: m \in \omega\right\}$ be a base for $Y, \mathscr{B}_{m}$ discrete. Then $f^{\leftarrow} \mathscr{B}_{m}=\left\{f^{\leftarrow B}: B \in \mathscr{B}_{m}\right\}$ is a disjoint Borel additive family. By Lemma 2.8, there is an $\alpha_{m}<\omega_{1}$ such that $f \leftarrow \mathscr{B}_{m}$ is $\Sigma_{\alpha_{m}}$ additive. Let $\alpha=\sup \left\{\alpha_{m}: m \in \omega\right\}$. Then $f^{\leftarrow \mathscr{B}}$ is $\Sigma_{\alpha}$ additive, hence $f \stackrel{m}{\in} \Psi_{\alpha} X Y$. Similarly there is $\beta<\omega_{1}$ such that $f^{-1} \in \Psi_{\beta} Y X$. So $f$ is a generalized homeomorphism of type $(\alpha, \beta)$.

We now turn to examining whether any of the above results can be improved by strengthening the conclusion, or by weakening the hypothesis, in particular, by omitting the assumption of Proposition P.

We begin by ruling out some improvements on the Baire, Lebesgue-Hausdorff theorem, even in the case $X=\mathbf{R}, Y=\mathbf{R}$. First, (c) cannot imply (b) 
because there are $2^{c}$ functions satisfying (c) and only $c$ satisfying (c). Second, we cannot replace "residual" with "almost everywhere" because there are closed nowhere dense sets of positive measure.

Next, to see why $Y$ must be a Banach space, consider $X=\mathbf{R}, Y=\{0,1\}$ $\subseteq \mathrm{R}$. Every continuous function from $X$ to $Y$ is constant, so $\Phi_{\alpha} X Y=\Phi_{0} X Y$ for all $\alpha$. On the other hand, the characteristic function of an open, not closed set is in $\Psi_{1} X Y-\Psi_{0} X Y$. The definition of $\Phi^{*}$ eliminates this example, and, as Banach showed, allows proving a Lebesgue-Hausdorff theorem for arbitrary separable $Y$. Kuratowski [11] made a different suggestion which, he points out, reduces to the case where $Y$ is a Banach space.

Let us note that the inclusion $\Phi_{\alpha} X Y \subset \Psi_{\alpha} X Y$ can be proved in $\mathrm{ZFC}$ for all $X, Y$, and $\alpha$.

Now we construct a counterexample to Theorem 2.1 from a $Q$ set $X \subseteq \mathbf{R}$. Let $f$ be a one-to-one map from $X$ to a sufficiently large Banach space $Y$. Clearly $f \in \Psi_{1} X Y$ as every subset of $X$ is $\Sigma_{1} X$, and it is easy to show that every element of $\Phi_{\alpha} X Y$ has separable range. Thus $f \in \Psi_{1} X Y-\Phi_{1} X Y$.

Unfortunately, the above is not a counterexample to Theorem 2.2, as $\Phi_{1}^{*} X Y=\Psi_{1} X Y$ by definition. It is plausible to try to construct a counterexample from a $Q_{\alpha}$ set. We define a $Q_{\alpha}$ set to be an uncountable subset $X$ of R such that $\alpha$ is least such that $\mathscr{P}(X)=\Sigma_{\alpha} X$. For every $\alpha<\omega_{1}$, a model of set theory with a $Q_{\alpha+1}$ set can be construcuted by A. Miller's techniques [15]. Of course, if $X$ is a $Q_{\alpha}$ set, then $2^{\text {card } X}=c$.

It follows from a result of Kuratowski [12, p. 517] that if $X$ is a $Q$ set then (c) is vacuously true. Kunen has extended this to show that $Q_{\alpha}$ sets are first category in themselves. Condition (c) is not the conclusion in Baire's formulation of his theorem; rather it is an intermediate conclusion. Baire proved his category theorem to conclude from condition (c) that $T$ was dense in $X$.

We append a list of some of the many questions left open by this discussion.

Question 1. Is it consistent that $\Phi_{2}^{*} \neq \Psi_{2}$ ? Can a counterexample be constructed from any $Q_{2}$ set? From Miller's $Q_{2}$ sets?

Question 2. Can Theorem 2.1 be strengthened by replacing "residual" with "dense"?

The next question, recently raised by Michael and Namioka [14], and answered affirmatively by Fremlin, seems closely related.

Question 3. Suppose $f \mid H$ has at least one point of continuity for every closed subset $H$ of $X$. Is $f$ continuous on a dense subset of $X$ ?

The author knows of no counterexample to the other consequences of Proposition P.

Question 4. Can Theorems 2.3, 2.4, 2.5, 28, and 2.9 be proved in ZFC? (Miller [15] has the following partial result: if $X \subset \mathbf{R}$ and every subset of $X$ is 
Borel in $X$, then there is $\alpha<\omega_{1}$ such that every subset of $X$ is $\Sigma_{\alpha} X$.)

Question 5. Can "disjoint" be replaced by "point finite" in Theorems 2.6 and 2.8 (with or without Proposition P)?

Question 6. Can the first sentence be omitted in the hypothesis of Theorem 2.3 ?

3. Outline of the proof of Proposition P. We begin by discussing six properties a family of subsets of a space can have. The analytic additive (aa) and $\sigma$ discretely decomposable ( $\sigma \mathrm{dd})$ properties have already been defined in the Introduction. (When we say that $\mathcal{S}$ is aa, we mean that $\mathcal{S}$ is aa relative to the subspace $\cup \mathcal{S}$.)

Following Pol, let us call $\delta=\left\{S_{i}: i \in I\right\}$ a $d$ family if whenever $x_{i} \in S_{i}$ then $\left\{\left\{x_{i}\right\}: i \in I\right\}$ is $\sigma \mathrm{dd}$. Clearly a $\sigma \mathrm{dd}$ family is a $d$ family.

It will be convenient to define subfamily to be something different from a subset. Suppose $\mathcal{S}=\left\{S_{i}: i \in I\right\}$ and $\mathcal{H}=\left\{H_{j}: j \in J\right\}$. We say $\mathcal{H}$ is a subfamily of $\mathcal{S}$, and write $\mathscr{H} \subset \subset^{*} \mathcal{S}$, if $J \subset I$ and for all $j \in J, H_{j}=S_{j} \cap$ $\cup \mathcal{H}$. The point of this definition is that if $\mathcal{S}$ is aa and $\mathcal{H} \subset * \mathcal{S}$ then $\mathcal{H}$ is aa. Similarly subfamilies inherit properties odd and $d$.

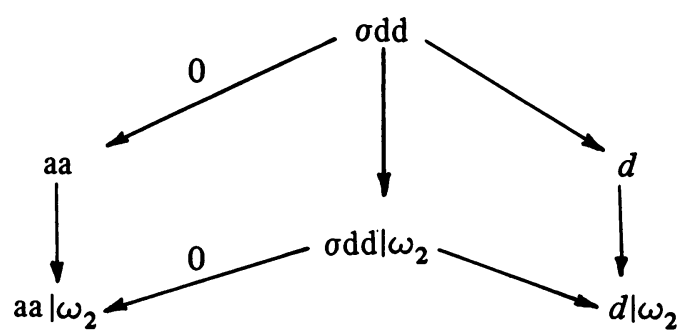

Diagram 1. Trivial implications

0 . Elements analytic

1. In general

2. $\forall S \diamond_{S}$, point-finite

3. ZFC

4. $S C \omega_{2}$, ultrametric

5. $V=L$, point-finite

6. $\exists \kappa E(\kappa)$

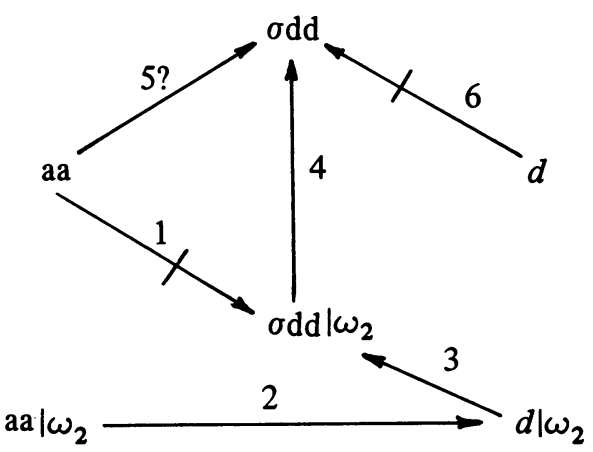

Diagram 2. Nontrivial implications 
Let $\nu$ be a cardinal. We say that $\delta$ is aa $\nu$ if whenever $\mathcal{H} \subset \subset^{*} \mathcal{S}$ and card $\cup \mathcal{H}<\nu$, then $\mathcal{H}$ is aa. We define $\sigma \mathrm{dd} \mid \nu$ and $d \mid \nu$ analogously.

Now we consider the implications between the six properties: aa, $\sigma \mathrm{dd}, d$, aa $\left|\omega_{2}, \sigma d d\right| \omega_{2}$, and $d \mid \omega_{2}$. There are six trivial implications, and these are displayed in Diagram 1. The downward arrows are because the properties are hereditary, the rightward arrows are because odd families are $d$ families, and the leftward arrows are from Hansell's converse with the additional hypothesis that the members of the family are analytic.

Diagram 2 displays some nontrivial implications. Of course, our main concern is the arrow from aa to odd. We start with some examples showing that aa does not imply even $\sigma \mathrm{dd} \mid \omega_{2}$ without additional hypotheses (arrow 1 ).

Let $X=\left\{x_{\alpha}: \alpha<\omega_{1}\right\}$ be a subset of $\mathbf{R}$. Let $S_{\beta}=\left\{x_{\alpha}: \beta<\alpha\right\}$. It is easy to verify that $\mathcal{S}=\left\{S_{\beta}: \beta<\omega_{1}\right\}$ is point countable, $\Pi_{1} X$ additive, and not $\sigma$ dd. By assuming the Continuum Hypothesis, we can make $\mathcal{S}$ absolutely $\Pi_{1}$ additive by letting $X=\mathbf{R}[\mathbf{1 0}]$.

To get an absolutely $\Pi_{1}$ additive not $\sigma$ dd example without the Continuum Hypothesis, let $X$ be the space of functions from $\omega$ to $\omega_{1}$, with the distance between two points $x, y$ defined to be $2^{-n}$, where $n$ is the least integer such that $x|n \neq y| n$. $X$ is complete. Define $S_{\beta}=\{x: \exists n x(n)>\beta\}$. Again it is easy to see that $\mathcal{S}=\left\{S_{\beta}: \beta<\omega_{1}\right\}$ is point countable and absolutely $\Pi_{1}$ additive. That $\delta$ is not $\sigma$ dd follows from Lemma 4.6.

The above example shows that there is no hope of proving aa $\rightarrow$ odd without the additional hypothesis that the set is point finite. Moreover, a $Q$ set shows that an additional set theoretic hypothesis is needed, even for disjoint $\Pi_{1}$ additive decompositions.

Now we start on our journey from aa to $\sigma \mathrm{dd}$, adding hypotheses along the way. The downward arrow is trivial. Lemma 4.8 shows that arrow 2 holds for disjoint families assuming $\diamond$ for stationary sets (abbreviated $\forall S \diamond_{s}$ ). Recall that a subset of $\omega_{1}$ is $c u b$ if it is closed and unbounded, and that a subset of $\omega_{1}$ is stationary if it meets every cub set. $\forall S \diamond_{S}$ is the assertion that for every stationary $S \subset \omega_{1}$ there is a function $\Gamma_{S}$ with domain $S$, such that for all $Y \subset \omega_{1},\left\{\alpha: \Gamma_{s}(\alpha)=Y \cap \alpha\right\}$ is stationary.

Arrow 3, due to Pol [18], holds in ZFC (cf. Lemma 4.6). The author independently proved an analogous lemma while seeking a nonmetrizable normal Moore space.

Lemma 4.9 shows that arrow 4 holds for certain metrizable subspaces, ultrametric spaces, under a new axiom of set theory $S C \omega_{2} . S C \omega_{2}$, whose complicated statement we defer, roughly says that $\omega_{2}$ retains a few properties of a supercompact cardinal.

One drawback to our proof of (the consistency of) Proposition $\mathbf{P}$ is that it requires a supercompact cardinal, a rather large cardinal. The author has no 
evidence to affirm or deny that $V=L$ implies Propoisition P. So we have labelled arrow 5 with a question mark.

The situation with respect to arrow 6 and large cardinals is different. Let $E(\kappa)$ be an axiom stating that $\kappa$ is a regular cardinal greater than $\omega_{1}$ and that there is a stationary $E \subset \kappa$ of $\omega$ limits such that, for all $\alpha<\kappa, E \cap \alpha$ is not stationary in $\alpha$. Jensen showed that $V=L$ implies $E(\kappa)$ for all regular, not weakly compact cardinals greater than $\omega_{1}$. It seems (at least to the author) that $\neg E\left(\omega_{2}\right)$ requires a weakly compact cardinal and that $\forall \kappa \neg E(\kappa)$ requires a strongly compact cardinal. Returning to metrizable spaces, we note that $\exists \kappa E(\kappa)$ implies that there is a $d$ family that is not odd [19]. Thus we have marked arrow 6 a nonimplication.

We conclude this section with a sketch of the proof of Proposition $\mathbf{P}$ from $S C \omega_{2}+\forall S \diamond_{s}$. Of course the basic idea is to follow the arrows. But because of the extra hypotheses the arrows require, it is easier to prove the contrapositive. First, by Lemma 4.4, if there is a point finite aa not $\sigma \mathrm{dd}$ family there is such a family $\mathcal{S}$ of subsets of an ultrametric space.

Now we follow the arrows backwards. By Lemma $4.9 \delta$ is not $\sigma \mathrm{dd} \mid \omega_{2}$; by Lemma $4.6, \delta$ is not $d \mid \omega_{2}$. Lemma 4.7 gives us a disjoint, not $d \mid \omega_{2}$ subfamily $\mathcal{S}^{\prime}$ of $\delta$. Then by Lemma $4.8 \mathcal{S}^{\prime}$ is not aa $\mid \omega_{2}$, a fortiori, not aa, and hence $\delta$ is not aa. This completes the proof of the contrapositive of Proposition P.

4. Many lemmas. In this section we prove the lemmas used in the proof of Proposition P. The lemmas are presented roughly in order of set theoretic sophistication asked of the reader.

Our first goal is to show that if there is a counterexample to Proposition P, then there is a counterexample in an ultrametric space. $X$ is an ultrametric space if $X$ has a base $\mathscr{B}=\cup\left\{\mathscr{B}_{m}: m \in \omega\right\}$ where each $\mathscr{B}_{m+1}$ is a clopen cover of $X$ refining $\mathscr{B}_{m}$. A continuous function $f$ from $X$ onto $Y$ is perfect if $f^{\rightarrow} K$ is closed in $Y$ for all $K$ closed in $X$ and $f^{\leftarrow}\{y\}$ is compact for all $y \in Y$. A map $f: X \rightarrow Y$ is irreducible if for all closed $K \underset{f}{\subsetneq} X, f \rightarrow K \neq f \rightarrow X$.

\subsection{LEMMA. Every space is the irreducible perfect image of an ultrametric} space.

Proof. This is Corollary 2 to Theorem VI.12 of Nagata [17]. As van Douwen pointed out to us, there is a proof along the following lines. A hedgehog is a space composed of unit intervals attached at 0; a Cantor set hedgehog is a space composed of Cantor sets attached at 0 . Every space $X$ with metric $\rho$ can be embedded in the product of hedgehogs. $X$ has a $\sigma$ discrete base $\mathscr{B}=\cup\left\{\mathscr{G}_{m}: m \in \omega\right\}$ where the members of $\mathscr{B}_{m}=\left\{B_{j}^{m}\right.$ : $\left.j \in J_{m}\right\}$ are farther than $2 / N_{m}$ apart. Let $Y_{m}$ be the hedgehog $\cup\left\{[0,1]_{j}\right.$ : $\left.j \in J_{m}\right\}$. For $n \geqslant N_{m}$, define $e_{m, n}: X \rightarrow Y_{m}$ by 


$$
e_{m, n}(x)=\max \left\{0,\left(1-n \cdot \rho\left(x, B_{j}^{m}\right)\right)_{j}: j \in J\right\} \text {. }
$$

Define $e: X \rightarrow \Pi\left\{Y_{m}^{n}: n \geqslant N_{m}, m \in \omega\right\}$ by

$$
e(x)=\left\langle e_{m, n}(x): n \geqslant N_{m}, m \in \omega\right\rangle .
$$

Finally, the perfect map from the Cantor set to the unit interval induces a perfect map from products of Cantor set hedgehogs onto products of hedgehogs. It is easy to use Zorn's lemma to show that if $f: X \rightarrow Y$ is perfect, there is a closed subset $K$ of $X$ so that $f \mid K$ is perfect and irreducible.

4.2. LEMMA. Let $\mathcal{H}$ be a locally finite family in $X$, and f a perfect map from $X$ onto $Y$. Then $f^{\rightarrow} \mathcal{H}=\left\{f^{\rightarrow H}: H \in \mathcal{C}\right\}$ is locally finite.

Proof. Fix $y \in Y$. For each $x \in f^{\leftarrow}\{y\}$ there is an open set $U_{x}$ containing $x$ and meeting at most finitely elements of $\mathcal{H}$. Because $f^{\leftarrow}\{y\}$ is compact, finitely many $U_{x}$ 's cover $f^{\leftarrow}\{y\}$. Now $V$, the union of these finitely many $U_{x}$ 's, is open, contains $f^{\leftarrow}\{y\}$, and meets only finitely elements of $\mathcal{H}$. Because $X-V$ is closed, $Y-f \rightarrow(X-V)$ is an open set containing $y$ and meeting at most finitely many elements of $f \rightarrow \mathcal{H}$.

4.3. LEMMA. Let $f$ be a perfect map from $X$ onto $Y$, and $\mathcal{S}$ a family of subsets of $Y$ :

(a) if $\mathcal{S}$ is $\Sigma_{\alpha}$ additive, analytic additive, $\sigma$ discretely decomposable, or a $d$ family, then so is $f \leftarrow \mathcal{S}$;

(b) if $\mathcal{S}$ is not $\sigma$ discretely decomposable, then neither is $f^{\leftarrow \mathcal{S} \text {; }}$

(c) if $f$ is irreducible and $\mathcal{S}$ is Baire property additive, then so is $f^{\leftarrow} \mathcal{S}$.

Proof. (a) follows routinely from the fact that the inverse image of an open set is open. (b) follows from the converse of Lemma 4.2 and the fact that because of the $\sigma$ discrete base, a locally finite family is $\sigma \mathrm{dd}$. (c) follows from the fact that the irreducible preimage of a nowhere dense set is nowhere dense. (A set $\delta$ has the Baire property if there is an open set $U$ such that $(U-S) \cup(S-U)$ is first category.)

4.4. Corollary. If there is a counterexample to Proposition $\mathrm{P}$ there is a counterexample in an ultrametric space.

Our second goal is to relate $\sigma \mathrm{dd} \mid \omega_{2}$ and $d \mid \omega_{2}$ to stationary subsets of $\omega_{1}$. For $I$ a set, $\nu$ a cardinal, let

$$
[I]^{<\nu}=\{J \subset I: \text { card } J<\nu\} .
$$

4.5. LeMMA. Let $\mathcal{S}=\left\{S_{i}: i \in I\right\}$ be a family of subsets of a space $X$ with $\sigma$ discrete base $\mathscr{B}=\cup\left\{\mathscr{B}_{m}: m \in \omega\right\}$. $\mathcal{S}$ is $\sigma$ discretely decomposable iff there is a function $\psi: \mathscr{B} \rightarrow[I]^{<\omega_{1}}$ satisfying

$$
\forall x \forall i, \quad x \in S_{i} \text { implies } i \in \cup\{\psi(B): x \in B\} .
$$


Proof. First we suppose there is such a function $\psi$. Let $\psi(B)=\{i(B, n)$ : $n \in \omega\}$. Then

$$
S_{i, m, n}=\left\{x \in S_{i}:\left(\exists B \in \mathscr{B}_{m}\right)(x \in B \text { and } i(B, n)=i)\right\}
$$

is a $\sigma$ discrete decomposition of $\mathfrak{S}$.

Conversely, let $\left\{S_{i, m}: i \in I, n \in \omega\right\}$ be a $\sigma$ discrete decomposition of $\delta$. Define

$$
\psi(B)=\left\{i: \exists n\left\{i: B \cap S_{i, n} \neq 0\right\}=\{i\}\right\} .
$$

The density $d(X)$ of a space $X$ is the least cardinal $\nu$ such that $X$ has a dense subset of cardinality $\nu$. Following Pol [18], [19], we call a disjoint family $\mathcal{H}=\left\{H_{\alpha}: \alpha<d(X)\right\}$ of subsets of $X$ a natural decomposition if, setting $X_{\beta}=\cup\left\{H_{\alpha}: \alpha \leqslant \beta\right\}$,

$$
\begin{gathered}
\forall \beta<d(X), \quad X_{\beta} \text { is closed, and } d\left(X_{\beta}\right)<d(X), \\
X=\cup\left\{H_{\alpha}: \alpha<d(X)\right\}=\cup\left\{X_{\beta}: \beta<d(X)\right\}, \\
\forall \text { limit } \lambda<d(X), \quad X_{\lambda}=\text { closure } \cup\left\{H_{\alpha}: \alpha<\lambda\right\} .
\end{gathered}
$$

4.6. LEMMA. Let $\mathcal{S}=\left\{S_{i}: i \in I\right\}$ be a family of subsets of a space $X$ of density $\omega_{1}$. Let $\mathcal{H}=\left\{H_{\alpha}: \alpha<\omega_{1}\right\}$ be a natural decomposition of $X$. Define $g$ : $I \rightarrow \omega_{1}$ by

$$
g(i)=\min \left\{\alpha: H_{\alpha} \cap S_{i} \neq \varnothing\right\} .
$$

Then the following are equivalent:

(a) $\mathcal{S}$ is $\sigma$ discretely decomposable,

(b) $\mathcal{S}$ is a d family,

(c) $g$ is countable-to-one and range $g$ is not stationary.

Proof. (a) $\rightarrow$ (b). Trivial.

(b) $\rightarrow$ (c). Let $x_{i} \in S_{i} \cap H_{g(i)}$. By (b), we may express $I=\bigcup\left\{I_{n}: n \in \omega\right\}$ so that each $Y_{n}=\left\{x_{i}: i \in I_{n}\right\}$ is discrete. Let $\mathscr{B}=\bigcup\left\{\mathscr{B}_{m}: m \in \omega\right\}$ be a $\sigma$ discrete base for $X$. Define $h: \mathscr{B} \rightarrow \omega_{1}$ by

$$
h(B)=\min \left\{\alpha: H_{\alpha} \cap B \neq \varnothing\right\} .
$$

Let $\left\{B_{i}: i \in I_{n}\right\} \subset \mathscr{B}$ be a disjoint family with $x_{i} \in B_{i}$. Define $k: I_{n} \rightarrow \omega_{1}$ by $k(i)=h\left(B_{i}\right)$. By (4.3) if $g(i)=\lambda$ is a limit ordinal, then $k(i)<g(i)$. Then the function defined by taking $g(i)$ to $k(i)$ is countable-to-one and presses down on limit ordinals. By the contrapositive of the pressing down lemma, range $g \mid I_{n}$ is not stationary. As the countable union of nonstationary sets is nonstationary, we conclude (c).

(c) $\rightarrow$ (a). By (c) there is a cub $C, C \cap$ range $g=\varnothing$. Let $\gamma(\alpha)=\sup \{C \cap$ $\alpha\} \in C$. Define $\psi(B)=\{i: \gamma(g(i))<h(B)\}$. We verify that $\psi$ is as in Lemma 4.5. Both $g$ and $\gamma$ are countable-to-one, so $\psi(B)$ is countable. Note that replacing $H_{\alpha}$ by $X_{\alpha}$ in the definition does not change $g$ and $h$. Because 
$C \cap$ range $g=\varnothing, \gamma(g(i))<g(i)$, and hence $S_{i} \cap X_{\gamma(g(i))}=\varnothing$. As $X_{\gamma(g(i))}$ is closed, for all $x \in S_{i}$ there is $B_{x} \in \mathscr{B}$ with $x \in B_{x}$ and $B_{x} \cap X_{\gamma(g(i))}=\varnothing$, so $i \in \psi\left(B_{x}\right)$.

REMARK. The above lemma is due to Pol [18, Theorem 1 and Remark 2]. The author independently proved an analogous lemma about Moore spaces $M(X, \delta)$ (Moore spaces may be nonmetrizable) constructed from $X$ and $\delta$ as in $[4, \S 3]$. This lemma is that $4.6(\mathrm{c})$ is equivalent to both

(d) $M(X, \delta)$ is metrizable,

(e) $M(X, \mathcal{S})$ is collectionwise Hausdorff.

One may further prove (a) $\leftrightarrow$ (d) and (b) $\leftrightarrow$ (e) without the assumption $d(X) \leqslant \omega_{1}$. The author is grateful for a letter from Roman Pol expressing this parallel and interesting the author in the subject of this paper.

4.7. Lemma. Suppose that $\mathcal{S}=\left\{S_{i}: i \in I\right\}$ is a point finite not $d$ family of subsets of $X, d(X) \leqslant \omega_{1}$. Then there is a disjoint, not $d$ family $\mathcal{S}^{\prime}$ of singletons, $\delta^{\prime} \subset^{*} \delta$.

Proof. Let $\mathcal{H}=\left\{H_{\alpha}: \alpha<\omega_{1}\right\}$ be a natural decomposition of $X$. Let $g$ : $I \rightarrow \omega_{1}$ be as in Lemma 4.6. Then there are two cases.

Case 1. For some $\delta<\omega_{1}, g^{\leftarrow}\{\delta\}$ is uncountable. For each $i \in g^{\leftarrow}\{\delta\}$ choose $x_{i} \in S_{i} \cap H_{\delta}$.

Let $F_{i}=\left\{x_{j}: j \in g^{\leftarrow}\{\delta\}\right.$ and $\left.x_{i} \in S_{j}\right\}$. Because each $F_{i}$ is finite, we may apply the $\Delta$-system lemma to get an uncountable $I^{\prime} \subset g^{\leftarrow}\{\delta\}$ such that $i, i^{\prime} \in I^{\prime}, i \neq i^{\prime}$ implies

$$
F_{i} \cap F_{i^{\prime}}=\cap\left\{F_{i}: i \in I^{\prime}\right\}
$$

and $x_{i} \notin \cap\left\{F_{i}: i \in I^{\prime}\right\}$. Let $S^{\prime}=\left\{\left\{x_{i}\right\}: i \in I^{\prime}\right\}$.

Case 2. Range $g$ is stationary. By restricting $I$, we may assume that $g$ is one-to-one onto $S$, a stationary set of limit ordinals. Choose $x_{i} \in S_{i} \cap H_{g(i)}$.

Let $\left\{F_{\alpha}: \alpha<\omega_{1}\right\}$ list the finite subsets of $\omega_{1}$ in such a manner that for every limit ordinal $\lambda<\omega_{1},\left\{F_{\alpha}: \alpha<\lambda\right\}$ lists the finite subsets of $\lambda$. For $\beta=g(i) \in S$, we define $h(\beta)$ so that

$$
F_{h(\beta)}=\left\{g(j): x_{i} \in S_{j}, g(j)<\beta\right\} .
$$

Since $h$ presses down on a stationary set, there is $\delta$ such that $h^{\leftarrow}\{\delta\}$ is stationary. Let $\mathcal{S}^{\prime}=\left\{\left\{x_{i}\right\}: h(g(i))=\delta\right\}$.

We now aim towards showing arrow 2 of Diagram 2. We begin by reviewing operation $Q$. Let $F S \omega$ be the set of finite sequences of integers. Given a function $U: F S \omega \rightarrow \mathscr{P}(X)$, we define

$$
Q(U)=\bigcup_{f \in \omega} \bigcap_{n \in \omega} U_{f \mid n} .
$$

We say a set $A$ is analytic in $X$ if there is a function $U$ from $F S \omega$ to the open sets of $X$ (equivalently, the closed sets of $X$ ) such that $A=\mathscr{Q}(U)$. We 
will find it convenient to define the analytic sets from basic open sets. So let $\mathscr{B}$ be a $\sigma$ discrete base for $X$. As every open set is the union of basic open sets, we may define the class of sets analytic in $X$ to be $\left\{\mathscr{Q}^{\prime}(u): u: \mathscr{B} \rightarrow\right.$ $\mathcal{P}(F S \omega)\}$, where

$$
Q^{\prime}(u)=\bigcup_{f \in \omega^{\omega} \omega} \bigcap_{n \in \omega} \bigcup_{f \mid n \in u(B)} B .
$$

Recall that $\diamond$ for stationary sets, abbreviated $\forall S \diamond_{S}$ is the assumption that whenever $S \subset \omega_{1}$ is stationary there is a function $\Gamma_{S}$ with domain $S$ such that for all $Y \subset \omega_{1},\left\{\alpha: \Gamma_{S}(\alpha)=Y \cap \alpha\right\}$ is stationary. Assuming that $d(X)<\omega_{1}$, letting $\left\{B_{\alpha}: \alpha<\omega_{1}\right\}=\mathscr{B}$ be a $\sigma$-discrete base, recalling that $\forall S \diamond_{S}$ implies the Continuum Hypothesis, and doing some bookkeeping, we can and do use the following variation of $\forall S \diamond_{s}$, convenient for dealing with operation $\mathbb{Q}$ : whenever $S \subset \omega_{1}$ is stationary there is a function $\Gamma_{S}$ with domain $S$ such that for all $u: \mathscr{B} \rightarrow \mathscr{P}(F S \omega),\left\{\alpha: \Gamma_{S}(\alpha)=u \mid \alpha\right\}$ is stationary.

4.8. LEMMA $\left(\forall S \diamond_{s}\right)$. If $d(X)<\omega_{1}$ and $\delta$ is an analytic additive disjoint family of subsets of $X$, then $\mathcal{S}$ is a d family; equivalently, $\mathcal{S}$ is $\sigma$ discretely decomposable.

Proof. We prove the contrapositive. So let $\mathcal{S}$ be not a $d$ family. Let $\mathcal{H}=\left\{H_{\alpha}: \alpha<\omega_{1}\right\}$ be the natural decomposition induced by setting

$$
X_{\alpha}=\left\{x \in X: \forall \gamma x \in B_{\gamma} \rightarrow \gamma<\alpha\right\} .
$$

By Lemma 4.6, the function $g$ is either uncountable-to-one or range $g$ is stationary. In the former case $\delta$ must be uncountable, and as a separable space has only $2^{\omega}$ analytic subsets, $2^{\omega}<2^{\omega_{1}}$ yields that $\delta$ is not aa.

In the latter case, we may assume that $\mathcal{S}$ is of the form $\left\{\left\{x_{\alpha}\right\}: \alpha \in S\right\}$ where $x_{\alpha} \in H_{\alpha}$ and $S$ is stationary. The key fact that allows us to use $\forall S \diamond_{S}$ is that

$$
\text { if } u\left|\alpha=u^{\prime}\right| \alpha \text { then } x_{\alpha} \in \mathbb{Q}^{\prime}(u) \text { iff } x_{\alpha} \in \mathbb{Q}^{\prime}\left(u^{\prime}\right) \text {. }
$$

Thus we may define $x_{\alpha} \in \mathbb{Q}^{\prime}(v)$, for $v$ from $\left\{B_{\beta}: \beta<\alpha\right\}$ to $\mathscr{P}(F S \omega)$ to mean $x_{\alpha} \in Q^{\prime}(u)$ for one, or equivalently, for all $u$ extending $v$.

Let $\Gamma_{S}$ be the function $\forall S \diamond_{S}$ asserts to exist. Define $Y \subset \omega_{1}$ by $Y=\{\alpha$ : $\left.x_{\alpha} \notin \mathbb{Q}^{\prime}\left(\Gamma_{S}(\alpha)\right)\right\}$.

We check that $Z=\left\{x_{\alpha}: \alpha \in Y\right\}$ is not analytic, i.e., $Z \neq \mathbb{Q}^{\prime}(u)$ for all $u$. Fix $u$. By our choice of $\Gamma_{S}$, there is an $\alpha \in S$ such that $u \mid \alpha=\Gamma_{S}(\alpha)$. Then $x_{\alpha} \in \mathbb{Q}(u)$ just in case $x_{\alpha} \notin Z$.

Our final goal is to demonstrate arrow 4 of Diagram 2. Axiom $S C \omega_{2}$ is rather technical, so for expository purposes, we precede its formulation with two applications.

Application 1. Let $\delta=\left\{S_{i}: i \in I\right\}$ be a family of subsets of an ultrametric space $X$, with base $\mathscr{B}=\bigcup\left\{\mathscr{B}_{m}: m \in \omega\right\}$ where each $\mathscr{B}_{m+1}$ is a clopen cover 
of $X$ refining $\mathscr{B}_{m}$. The reason we have considered ultrametric spaces is that we need (at least, the author needs) to define a tree $T$, <. Let $T=\mathscr{B} \cup$ $\{\{x\}: x \in X\}$; define $t^{\prime} \leqslant t$ iff $t^{\prime} \supset t$. Define $\bar{t}=\left\{t^{\prime}: t^{\prime}<t\right\}$.

Now Lemma 4.5 says that $\delta$ is $\sigma$ dd iff there is a $\psi: \mathscr{B} \rightarrow[I]^{<\omega_{1}}$ satisfying

$$
\forall x \forall i, \quad x \in S_{i} \text { implies } i \in \cup\{\psi(B): x \in B\} .
$$

Translating this into tree terminology

$$
\forall t \in X, \quad \forall i \in I, \quad t \in S_{i} \text { implies } i \in \cup\left\{\psi\left(t^{\prime}\right): t^{\prime} \in \bar{t}\right\} .
$$

Next, we note that whether $\psi$ meets the condition at $t$ depends on only part of the information contained in $\psi$. Only the restriction of $\psi$ to $t$ and the set $\psi\left(t^{\prime}\right) \cap\left\{i: t \in S_{i}\right\}$ are relevant. We indicate this by rephrasing our expression:

$$
\forall t \in X, \quad\left\{i: t \in S_{i}\right\} \subset \cup\left\{\psi \mid \bar{t}\left(t^{\prime}\right) \cap\left\{i: t \in S_{i}\right\}: t^{\prime} \in \bar{t}\right\} .
$$

Now $S C \omega_{2}$ will say that if for all $T^{\prime} \in[T]^{<\omega_{2}}$ there is a $\psi_{T^{\prime}}$ meeting such a condition at all $t \in T^{\prime}$, there is a $\psi$ which meets the condition at all $t \in T$. Thus, we prove Lemma 4.9 (below).

Application 2. Let $T$, $<$ be a tree of height $<\omega_{1}$. That is, for all $t \in T$, $\bar{t}=\left\{t^{\prime}: t^{\prime}<t\right\}$ is countable and well ordered by $<$. We topologize $T$ by declaring sets of the form $\{t \in T: a<t \leqslant b\}$ and $\left\{\{t\}:\left(\neg \exists t^{\prime}\right)\left(t^{\prime}<t\right)\right\}$ to be basic open. $T$ is a regular, first countable, locally countable, locally compact Moore, possibly nonmetrizable space.

A space is collectionwise Hausdorff (respectively $\omega_{2}$ collectionwise Hausdorff) if every closed discrete set (respectively, of cardinality $<\omega_{2}$ ) can be screened (i.e., simultaneously separated by disjoint open sets).

Let $Y$ be a closed discrete set of nonisolated points of $T . \quad Y$ can be screened iff there is a function $F$ from $T$ to $[Y]^{<\omega_{1}}$ such that for all $y$ there is $t<y$ such that for all $t^{\prime}, t \leqslant t^{\prime}<y$ implies $F\left(t^{\prime}\right)=\{y\}$.

As in Application 1, whether $F$ meets this condition at $y$ depends only on part of the information contained in $F$. We may rephrase the condition as: for all $y \in Y$ there is a $t<y$ such that for all $t^{\prime}, t<t^{\prime}<y$ implies $F \mid \bar{y}\left(t^{\prime}\right) \cap\{y\}=\{y\}$. Thus, we prove Lemma 4.10 (below).

(It happens that in this particular case we can improve a "point countable almost screening" to a screening. But for convenience in this case, and perhaps necessity in some other case, we include in $S C \omega_{2}$ a provision to make $F(t)$ a singleton.)

$S C \omega_{2}$ will say that if for all $Y^{\prime} \in[Y]^{<\omega_{2}}$ there is an $F_{Y^{\prime}}$ meeting such a condition at all $y \in Y^{\prime}$ there is an $F$ meeting the condition at all $y \in Y$.

We introduce notation and terminology to express $S C \omega_{2}$. We call a hextuple $\mathcal{T}=\langle T,\langle, A, \delta, z, \theta\rangle$ acceptable if

(a) $\left\langle T,\langle\rangle\right.$ is a tree of height $\leqslant \omega_{1}$, 
(b) $A$ is a set,

(c) $\delta, z$, and $\theta$ are functions with domain $T$ such that for all $t \in T$ :

(1) $\delta(t) \in[A]^{<\omega_{2}}$

(2) $z(t)$ is a set of functions from $\bar{t}$ to $[A]^{<\omega_{1}}$,

(3) $\theta(t)$ is a function from $\bar{t}$ to $\omega+1$.

If $T^{\prime} \subset T$, set $T^{\prime \prime}=\cup\left\{\bar{t}: t \in T^{\prime}\right\}$ and

$$
\mathcal{T}^{\prime}=\left\langle T^{\prime \prime},\left\langle\left|T^{\prime \prime} \times T^{\prime \prime}, A, \delta\right| T^{\prime \prime}, z\left|T^{\prime \prime}, \theta\right| T^{\prime \prime}\right\rangle .\right.
$$

Set

$$
[f, t, \delta]=\left\{\left\langle t^{\prime}, f\left(t^{\prime}\right) \cap \delta(t)\right\rangle: t^{\prime} \in \bar{t}\right\} .
$$

We call an acceptable hextuple $\mathcal{T}$ doable if there is a function $f: T \rightarrow[A]^{<\omega_{1}}$ such that for all $t \in T$

(a) $[f, t, \delta] \in z(t)$,

(b) for all $t^{\prime} \in \bar{t}$, card $f\left(t^{\prime}\right) \leqslant \theta(t)\left(t^{\prime}\right)$.

In such case we say $f$ does $\mathcal{T}$. We say that $\mathcal{T}$ is $\omega_{2}$ doable if for all $T^{\prime} \in[T]^{<\omega_{2}}, \mathcal{T}^{\prime}$ is doable.

Axiom $S C \omega_{2}$ is the assertion that an acceptable hextuple $\mathcal{T}$ is doable iff $\mathcal{T}$ is $\omega_{2}$ doable.

We remark that one can modify $S C \omega_{2}$ to $S C \nu^{++}$by changing $\omega$ to $\nu, \omega_{1}$ to $\nu^{+}$, and $\omega_{2}$ to $\nu^{++}$throughout the above discussion and in the proof that $S C \omega_{2}$ holds in a model obtained by Levy collapsing a supercompact cardinal.

4.9. LEMMA. $\left(S C \omega_{2}\right)$ let $\mathcal{S}$ be a family of subsets of an ultrametric space $X$. $\mathcal{S}$ is $\sigma$ discretely decomposable if $\mathcal{S}$ is $\sigma d d \mid \omega_{2}$.

Proof. Set $T=\mathscr{B} \cup\{\{x\}: x \in X\}, \quad<=\supset, \quad A=I$,

$$
\delta(x)=\left\{i: x \in S_{i}\right\}, \quad \delta(B)=\varnothing,
$$

$z(x)=\left\{f: f\right.$ is a function from $\bar{x}$ to $[I]^{<\omega_{1}}, \delta(x) \subseteq \cup$ range $\left.f\right\}$,

$z(B)=\left\{f: f\right.$ is a function from $\bar{B}$ to $\left.[I]^{<\omega_{1}}\right\}, \theta(t)\left(t^{\prime}\right)=\omega$.

4.10. LEMMA. $\left(S C \omega_{2}\right)$ A tree space $T,<$ of height $<\omega_{1}$ is collectionwise Hausdorff if $\omega_{2}$ collectionwise Hausdorff.

Proof. Let $Y$ be a closed discrete subset of $T$. Set $A=Y$; for $y \in Y$, $\delta(y)=\{y\}$,

$$
\begin{aligned}
& z(y)=\left\{f: f \text { is a function from } \bar{y} \text { to }[Y]^{<\omega_{1}},\right. \\
& \left.\qquad \exists t<y, \forall t^{\prime}, t<t^{\prime}<y \rightarrow f\left(t^{\prime}\right)=\{y\}\right\},
\end{aligned}
$$


$\theta(y)\left(t^{\prime}\right)=1$; for $t \notin Y, \delta(t)=\varnothing$,

$$
z(t)=\left\{f: f \text { is a function from } \bar{t} \text { to }[Y]^{<\omega_{1}}\right\}, \theta(t)\left(t^{\prime}\right)=1 .
$$

We remark that there are counterexamples to Lemmas 4.9 and 4.10 assuming $\exists \kappa E(\kappa)$.

It would be nice to have other applications of $S C \omega_{2}$ or, more likely, strengthen $S C \omega_{2}$ to have other applications. Some candidates are

(1) a graph has chromatic number $\leqslant \omega$ iff every subgraph of cardinality $<\omega_{2}$ has chromatic number $\leqslant \omega$;

(2) a first countable space is metrizable iff every subspace of cardinality $<\omega_{2}$ is metrizable;

(3) Application 2 with " $T$ is a tree space of height $\omega_{1}$ " replaced with " $T$ is first countable".

Shelah [22, p. 322] has claimed (1) with "chromatic" replaced by "coloring". In seeking applications of this type it is prudent to note that there is a family of $\omega_{2}$ countable sets without a transversal, yet every subfamily of cardinality $<\omega_{2}$ has a transversal [16].

5. Collapsing a supercompact cardinal to $\omega_{2}$. We begin by defining the partial order of Lévy forcing. For ordinals $\lambda<\nu$, set $[\lambda, \nu)=\{\rho: \lambda<\rho<\nu\}$. For $\omega_{2} \leqslant \lambda<\nu$, let $P(\lambda, \nu)$ be the set of $p$ satisfying (5.1)-(5.4) below, with the partial order given by (5.5):

$$
\begin{gathered}
p \text { is countable, } \\
p \text { is a function, } \\
\text { domain } \rho \subset[\lambda, \nu) \times \omega_{1}, \\
p(\rho, \alpha) \in \rho, \\
p^{\prime} \leqslant p \text { iff } p^{\prime} \supseteq p .
\end{gathered}
$$

It is obvious that $P(\lambda, \nu)$ is countably closed. So $\omega_{1}$ is preserved and we omit superscripts. If $\nu$ is inaccessible, $P(\lambda, \nu)$ has the $\nu$ chain condition (apply the $\Delta$-system lemma to the domains of the $p$ 's). In this case $\nu$ becomes $\omega_{2}$ in the extension. Also note that when $\lambda<\kappa<\nu, P(\lambda, \nu)=P(\lambda, \kappa) \oplus P(\kappa, \nu)$.

5.1. LemMA. Let $\kappa$ be inaccessible and let $G$ be $M$ generic over $P\left(\omega_{2}, \kappa\right)$. Then $\diamond$ for stationary sets holds in $M[G]$.

Proof. Because $\kappa$ is inaccessible $2^{\omega}<\kappa$. List $\cup\left\{\mathscr{P}(\alpha): \alpha<\omega_{1}\right\}$ as $\left\{a_{\beta}\right.$ : $\left.\beta<2^{\omega}\right\}$.

Suppose that $M[G] \vDash S \subset \omega_{1}$ is stationary. By the $\kappa$ chain condition, there is a $\nu, 2^{\omega} \leqslant \nu<\kappa$, such that $M\left[G \cap P\left(\omega_{2}, \nu\right)\right] \vDash S \subset \omega_{1}$ is stationary. Define, in $M[G]$, a function $\Gamma$ with domain $S$ by $\Gamma(\gamma)=a_{\cup G(\nu, \gamma)}$.

Working in $M\left[G \cap P\left(\omega_{2}, \nu\right)\right]$, we verify that $\Gamma$ witnesses $\diamond$ for $S$. Suppose 
$p \in P(\nu, \kappa), p \Vdash C^{0}, Y^{0} \subset \omega_{1}, C^{0}$ cub. We will find $p^{\prime}<p$ and $\gamma<\omega_{1}$ so that $p^{\prime} \mathbb{H} \gamma \in C^{0} \cap S, \Gamma(\gamma)=Y^{0} \cap \gamma$. Using the facts that $\omega_{1}$ is regular and that $P(\nu, \kappa)$ is countable closed, we can define by induction on $\alpha, \alpha<\omega_{1}$, conditions $p_{\alpha} \in P(\nu, \kappa)$, ordinals $\beta_{\alpha}<\omega_{1}$, and sets $Y_{\alpha} \subset \beta_{\alpha}$ satisfying for all $\alpha^{\prime}<\alpha$ and all limit ordinals $\lambda$,

$$
\begin{aligned}
& p>p_{\alpha^{\prime}}>p_{\alpha}, \quad \beta_{\alpha^{\prime}}<\beta_{\alpha}, \\
& p_{\lambda}=\bigcup\left\{p_{\alpha}: \alpha<\lambda\right\}, \quad \beta_{\lambda}=\bigcup\left\{\beta_{\alpha}: \alpha<\lambda\right\}, \\
& p_{\alpha} \Vdash C^{0} \cap\left[\beta_{\alpha^{\prime}}, \beta_{\alpha}\right) \neq \varnothing, \\
& p_{\alpha} \Vdash Y^{0} \cap \beta_{\alpha}=Y_{\alpha} \text {, } \\
& (\nu, \delta) \in \operatorname{dom} p_{\alpha} \rightarrow \delta<\beta_{\alpha} \text {. }
\end{aligned}
$$

Because $\left\{\beta_{\alpha}: \alpha<\omega_{1}\right\}$ is cub and $S$ is stationary, there is a limit ordinal $\lambda$ so that $\beta_{\lambda} \in S$. Note that because $p \Vdash C^{0}$ cub, $p_{\lambda} \Vdash \beta_{\lambda} \in C^{0}$. Suppose $Y_{\lambda}=a_{\delta}$. Then

$$
p_{\lambda} \cup\{\langle\langle\nu, \lambda\rangle, \delta\rangle\} \Vdash \beta_{\lambda} \in C^{0} \cap S, \quad \Gamma\left(\beta_{\lambda}\right)=Y^{0} \cap \beta_{\lambda} .
$$

A cardinal $\kappa$ is supercompact iff for all $\lambda>\kappa$ there is a transitive class $N$ and an elementary embedding $J: V \rightarrow N$ satisfying (we will write $j x$ instead of $j(x)$ )

$$
\begin{gathered}
j|\kappa=\mathrm{id}| \kappa, \\
j \kappa>\lambda, \\
{ }^{\lambda} N \subset N .
\end{gathered}
$$

The standard reference on supercompact cardinals is [21].

Let $\mathscr{B}$ be the regular open algebra of $P\left(\omega_{2}, \kappa\right)$. Then $j$, as above, is a complete Boolean algebra embedding of $\mathscr{B}$ into $j \mathscr{B}$, which is the regular open algebra of $P\left(\omega_{2}, j \kappa\right)$. This induces an elementary embedding, also denoted $j$, of the Boolean valued model $M^{\mathscr{B}}$ into $N^{j \mathscr{B}}$. An important fact about this embedding follows from (5.6).

5.2. Lemma. If $x \in M^{\mathscr{B}}$ and card $x<\kappa$, then $j x=\{j y: y \in x\}$.

5.3. THEOREM. Let $\kappa$ be a supercompact cardinal in a model M. Then $S C \omega_{2}$ is valid in $\boldsymbol{M}^{\mathscr{B}}$.

Proof. We outline the proof, supplying the reasons later. Suppose

$$
M \vDash \| \mathcal{T} \text { is } \omega_{2} \text { doable } \|^{\circledR}=b .
$$

Choose an elementary embedding $j$ with $\lambda$ large enough that (5.8) gives $\mathcal{T} \in N$ and (5.7) gives card $T<j \kappa$.

$$
\begin{gathered}
N \vDash \| j \mathcal{T} \text { is } j \kappa \text { doable } \|^{j \Phi}>j b, \\
N \vDash \| \mathcal{T} \text { is doable } \|^{j \mathscr{B}}>j b,
\end{gathered}
$$




$$
\begin{aligned}
& N \vDash \| \mathcal{T} \text { is doable } \|^{\mathscr{B}} \geqslant b, \\
& M \vDash \| \mathcal{T} \text { is doable } \|^{\mathscr{B}} \geqslant b .
\end{aligned}
$$

First, (5.10) follows from (5.9) because $j$ is elementary, recalling that $\kappa$ is $\omega_{2}$ in $M^{\mathscr{B}}$. We chose $j$ so that $\mathcal{T} \in N$, thus (5.11) is meaningful. If $T \subset j T$ and $A=j A$, then (5.11) follows from (5.10) because we chose $j$ so that card $T<j \kappa$. While $T \subset j T$ and $A=j A$ need not hold, we will show in Lemma 5.4 below that this basic idea works. That (5.12) follows from (5.11) boils down to Lemma 5.5 , that "not doable" is preserved by countably closed forcing, in particular $P(\kappa, j \kappa)$. Since $N^{\mathscr{B}} \subset M^{\mathscr{B}}$ and $\mathcal{T}$ is doable is a $\Sigma_{1}$ formula, (5.12) gives (5.13). This derivation of (5.13) from (5.9) shows that $S C \omega_{2}$ is valid in $M^{\mathscr{B}}$.

5.4. LeMMA. $N \vDash j \mathcal{T}$ is $j \kappa$ doable $\left\|^{j \mathscr{B}} \leqslant\right\| \mathcal{T}$ is doable $\|^{j \mathscr{B}}$.

Proof. As pointed out above, if $T \subset j T$ and $j A=A$, the lemma is obvious. The idea of this proof is to note that there is a subset of $j T$ which is a copy of $T$, and that because of card $\delta(t)<\omega_{2}, j A-A$ is irrelevant.

Define $T^{*}$ by (omiting the superscript $j \Re$ )

$$
\left\|x \in T^{*}\right\|=\|(\exists t \in T)(x=j t)\| \text {. }
$$

(We check that

$$
\|(\exists t \in T)(x=j t)\| \cdot\|x=y\| \leqslant\|(\exists t \in T)(y=j t)\|
$$

so the definition of $T^{*}$ is valid.) Now card $T^{*}<j \kappa$ and $T^{*} \subset j T$, so there is $g: T^{*} \rightarrow[j A]^{<\omega_{1}}$ with

$$
\| g \text { does } T^{*}\|\geqslant\| j \mathcal{T} \text { is } j \kappa \text { doable } \| .
$$

Define $A^{*} \subset j A, h: T^{*} \rightarrow\left[A^{*}\right]^{<\omega_{1}}$, and $f: T \rightarrow[A]^{<\omega_{1}}$ by

$$
\begin{aligned}
\left\|x \in A^{*}\right\| & =\|(\exists a \in A)(x=j a)\|, \\
\|x \in h(t)\| & =\|x \in g(t)\| \cdot\left\|x \in A^{*}\right\|, \\
\|a \in f(t)\| & =\|j a \in g(j t)\| .
\end{aligned}
$$

We claim that

$$
\| g \text { does } \mathcal{T}^{*}\|=\| h \text { does } \mathcal{T}^{*}\|\leqslant\| f \text { does } \mathcal{T} \| .
$$

First we note that for all $t \in T$, $\operatorname{card} g(j t) \geqslant \operatorname{card} h(j t)=\operatorname{card} f(t)$. Thus we verify that range $h \subset\left[A^{*}\right]^{<\omega_{1}}$, range $f \subset[A]^{<\omega_{1}}, h$ satisfies (4.5b) and $f$ satisfies (4.5b). Next we see that by Lemma 5.2, for all $t \in T, j \delta(j t)=\{j a$ : $a \in \delta(t)\} \subset A^{*}$. So $[g, j t, j \delta]=[h, j t, j \delta]=j[f, t, \delta]$, hence

$$
\begin{aligned}
\|[g, j t, j \delta] \in j z(j t)\| & =\|[h, j t, j \delta] \in j z(j t)\| \\
& =\|[f, t, \delta] \in z(t)\| .
\end{aligned}
$$

5.5. LemMA. Suppose that in $M, P$ is countably closed and $\mathcal{T}$ is an acceptable tuple. Let $G$ be $M$ generic over $P$. If $\mathcal{T}$ is doable in $M[G]$, then $\mathcal{T}$ is doable in M. 
Proof. By the basic forcing lemmas, if $\mathcal{T}$ is doable in $M[G]$, there is a condition $p \in G$ and a term $f^{0}$ such that $p \Vdash f^{0}$ does $\mathcal{T}$. Note that because $P$ is countably closed $[A]^{<\omega_{1}}$ is the same in both $M$ and $M[G]$. Thus in $M$ we may define by induction on $<\sigma$ conditions $p_{t} \in P$ and elements $f(t)$ of $M$ for all $t \in T$ such that

$$
\begin{gathered}
p_{t} \leqslant p p \\
t^{\prime}<\tau t \text { implies } p_{t^{\prime}} \geqslant p p_{t},
\end{gathered}
$$

and

$$
p_{t} \Vdash f^{0}(t)=f(t)
$$

For all $t \in T p_{t} \Vdash f^{0}|\bar{t}=f| \bar{t} \wedge f^{0}$ does $\mathcal{T}$. Thus $f \in M$ does $\mathcal{T}$ and $\mathcal{T}$ is doable in $M$.

\section{BIBLIOGRAPHY}

1. R. Baire, Leçons sur les fonctions discontinues, Gauthier-Villars, Paris, 1905.

2. S. Banach, Uber analytisch darstellbare Operationen in abstrakten Raumen, Fund. Math. 17 (1931), 283-295.

3. J. Baumgartner, Some new order types, Ann. Math. Logic 9 (1976), 187-222.

4. W. Fleissner, Separation properties in Moore spaces, Fund. Math. 98 (1978), 279-286.

5. R. Hansell, Borel measurable mappings for nonseparable metric spaces, Trans. Amer. Math. Soc. 161 (1971), 145-169. MR 44 \#5426.

6. On Borel mappings and Baire functions, Trans. Amer. Math. Soc. 194 (1974), 195-211.

7. F. Hausdorff, Mengenlehre, de Gruyter, Berlin, 1937; English translation, Chelsea, New York, 1957. MR 19, 11.

8. M. Lavrentiev, Sur la recherche des ensembles homéomorphes, C. R. Acad. Sci. Paris 178 (1924), 187.

9. H. Lebesgue, Sur les fonctions représentables analytiquement, J. Math. Pures Appl. 1 (1905), 139-216.

10. J. Kaniewski and R. Pol, Borel-measurable selectors for compact-valued mappings in the nonseparable case, Bull. Acad. Polon. Sci. Sér. Sci. Math. Astronom. Phys. 23 (1975), 1043-1050.

11. K. Kuratowski, Quelques problèmes concernant espaces métriques non-séparables, Fund. Math. 25 (1935), 532-545.

12. MR 36 \#840.

13. A. Martin and R. Solovay, Internal Cohen extensions, Ann. Math. Logic 2 (1970), 143-178.

14. E. Michael and I. Namioka, Barely continuous functions, Bull. Acad. Polon. Sci. Sér. Sci. Math. Astronom. Phys. 24 (1976), 889-892.

15. A. Miller, On the length of Borel hierarchies. (to appear).

16. E. Milnor and S. Shelah, Some theorems on transversals, Colloq-Math. Soc. János Bolyai 10 (1973), 1115-1126.

17. J. Nagata, Modern general topology, North Holland, Amsterdam, 1968.

18. R. Pol, Note on decompasitions of metrizable spaces, Fund. Math. 95 (1977), 95-103.

19. $\ldots$ Note on decompasitions of metrizable spaces. II, Fund. Math. 100 (1978), 129-143.

20. D. Preiss, Completely additive disjoint systems of Baire sets is of bounded class, Comment. Math. Univ. Carolinae 15 (1972), 341-344. 
21. A. Kanamori, W. Reinhardt and R. Solovay, Strong axioms of infinity and elementary embeddings, Ann. Math. Logic 13 (1978), 73-116.

22. S. Shelah, A complactness theorem for singular cardinals, free alsebras, Whitehead problem, and transwersals, Israel J. Math. 21 (1975), 319-349.

23. R. Solovay and S. Tennenbaum, Iterated Cohen extensions and Souslin's problem, Ann. of Math. (2) 94 (1971), 201-245.

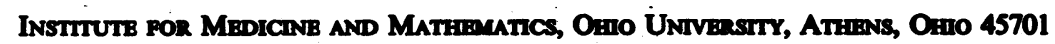

Current address: Department of Mathematics, University of Pittoburgh, Pittsburgh, Penngylvania 15260 